\title{
NOISE FILTERING METHOD FOR COLOR IMAGES BASED ON LDA AND NONLINEAR DIFFUSION
}

\author{
Woong Hee Kim and Thomas Sikora \\ Communication Systems Group \\ Technical University of Berlin \\ \{whkim, sikora\}@ nue.tu-berlin.de
}

\begin{abstract}
The purpose of noise filtering for images is to preserve features such as edge or corners in images, while reducing noise. Recent noise filtering algorithms based on diffusion equation shows the satisfactory results to some extent, if the noise is additive Gaussian noise. However, if the noise is not additive Gaussian noise, the filtering result is not satisfactory. In this paper, we propose a noise filtering method for color images based on LDA and nonlinear diffusion, which makes use of a common diffusion control. Experimental results with images degraded by additive Gaussian noise, salt and pepper noise, and multiplicative noise are presented.
\end{abstract}

Index Terms - Noise Filtering, Nonlinear Diffusion, Linear Discriminant Analysis

\section{INTRODUCTION}

Many noise filtering methods for images using diffusion equation have been proposed in recent years [1, 2, 3, 6, 7]. Perona and Malik made a significant contribution in the area of noise filtering with diffusion equation [1]. They proposed a nonlinear diffusion algorithm for noise filtering of images. Most of the noise filtering methods using diffusion equation have a diffusion control term, which is based on the gradient information of an initial image or successive filtered images by iteration. The diffusion control term decreases the diffusivity near the image features like edge or corners, and increases it in homogeneous region without them. The performance of filtering algorithms depends on the edge detector to some extent. The traditional noise filtering algorithms of diffusion equation use a simple edge detection method based on gradient of pixel values. These kinds of diffusion control terms show the satisfactory results, if the image is degraded by additive Gaussian noise. However, the edge detection algorithm is sensitive to other kinds of noise such as salt and pepper noise.

In this paper, we present a noise filtering method for color images, which is based on the gLDA(generalized Linear Discriminant Analysis) [5] and diffusion equation. The edge map for diffusion control term is evaluated with the regenerated image space using gLDA, not successive filtered images. This paper is organized as follows. In section 2, the fundamentals of gLDA and the background and related works on noise filtering algorithms using diffusion equation are explained. Section 3 presents the proposed noise filtering algorithm. Section 4 shows the experimental results with images degraded by additive Gaussian noise, multiplicative noise, and Salt and Pepper noise. We compare our method with other noise filtering algorithms using diffusion equation. Finally, section 5 concludes the paper.

\section{LDA AND DIFFUSION EQUATION FOR NOISE FILTERING}

\subsection{LDA}

The goal of LDA(Linear Discriminant Analysis) is to find the direction for efficient separation of data. The within-class scatter matrix and the between-class scatter matrix determines the separation criterion as follows [4].

Scatter matrix is defined as

$$
\mathbf{S}_{\mathbf{i}}=\sum_{\mathbf{x} \in \mathbf{G}_{\mathbf{i}}}\left(\mathbf{x}-\mathbf{m}_{\mathbf{i}}\right)\left(\mathbf{x}-\mathbf{m}_{\mathbf{i}}\right)^{t}
$$

where $\mathbf{x}$ is a data in a group $\mathbf{G}_{\mathbf{i}}$, and $\mathbf{m}_{\mathbf{i}}$ means the centroid of the group $\mathbf{G}_{\mathbf{i}}$.

between-class scatter matrix $\left(\mathbf{S}_{\mathbf{B}}\right)$ and within-class scatter $\operatorname{matrix}\left(\mathbf{S}_{\mathbf{W}}\right)$ are defined respectively as follows.

$$
\begin{gathered}
\mathbf{S}_{\mathbf{B}}=\left(\mathbf{m}_{1}-\mathbf{m}_{\mathbf{2}}\right)\left(\mathbf{m}_{\mathbf{1}}-\mathbf{m}_{\mathbf{2}}\right)^{\mathbf{t}} \\
\mathbf{S}_{\mathbf{W}}=\mathbf{S}_{1}+\mathbf{S}_{\mathbf{2}}
\end{gathered}
$$

The solution to maximize the ratio between $\mathbf{S}_{\mathbf{B}}$ and $\mathbf{S}_{\mathbf{W}}$ is (4).

$$
\mathbf{w}=\mathbf{S}_{\mathbf{W}}^{-1}\left(\mathbf{m}_{1}-\mathbf{m}_{2}\right)
$$

In traditional LDA, $\mathbf{S}_{\mathbf{W}}$ should be nonsingular. To solve this problem, several methods are proposed [5]. We use regularized LDA for our noise filtering algorithm to overcome 
the singularity problem. This adds a scaled identity matrix to $\mathbf{S}_{\mathbf{W}}$, when $\mathbf{S}_{\mathbf{W}}$ is singular.

$$
\tilde{\mathbf{S}}_{\mathbf{W}}=\mathbf{S}_{\mathbf{W}}+\alpha \mathbf{I}
$$

where $\alpha>0$ and $\mathbf{I}$ means a identity matrix.

\subsection{Diffusion Equation for Noise Filtering}

Perona and Malik proposed a new diffusion equation (6) with the initial condition (7) [1].

$$
\begin{aligned}
& \frac{\partial u}{\partial t}=\nabla \cdot(g(|\nabla u|) \nabla u) \\
& u(x, y, t=0)=I(x, y)
\end{aligned}
$$

where $I(x, y)$ is a two-dimensional image, which maps $I: \mathbb{R}^{2} \rightarrow \mathbb{R}$.

In (6), $g(s)$ is a nonnegative monotonically decreasing function. For example,

$$
g(s)=e^{\left(-\frac{s^{2}}{K}\right)}
$$

or

$$
g(s)=\frac{1}{1+K s^{2}}
$$

where $K$ is a constant.

However, when we apply this method to reduce some noise, image features such as edge or corners are blurred, or noise still remains in the image. Weickert proposed the texture enhancement algorithm using structure tensor information [3]. The diffusivity control term does not depend on the scalar value, but vector quantity in that algorithm, which shows the better performance to enhance texture or coherence.

Alvarez, Lions, and Morel modified Perona-Malik diffusion equation (6) as follows.

$$
\frac{\partial u}{\partial t}=g\left(\left|\nabla G_{\sigma} * u\right|\right)|\nabla u| \nabla \cdot\left(\frac{\nabla u}{|\nabla u|}\right)
$$

where $G_{\sigma}$ is a convolution kernel with kernel size $\sigma$, and * means convolution operation.

Nordström [2] proposed a balancing term or regularization term in Perona-Milik's model (6), which is described by (9). The balancing term makes the filtered image close to the initial image.

$$
\frac{\partial u}{\partial t}-\nabla \cdot(g(\nabla u) \nabla u)=I-u
$$

Barcelos et al. [6] proposed a new parabolic equation for image restoration using a balancing term, which results in (10).

$$
\frac{\partial u}{\partial t}=g|\nabla u| \nabla \cdot\left(\frac{\nabla u}{|\nabla u|}\right)-\lambda(1-g)(u-I)
$$

where $g$ means $g\left(\left|\nabla G_{\sigma} * u\right|\right)$, and $\lambda$ is a constant. Also, $g\left(\left|\nabla G_{\sigma} * u\right|\right)$ means that the edge detection is done in Gaussian scale space, and the gradient values are filtered with Gaussian kernel to reduce the effect of noise. The diffusion control term of these diffusion equation is a function of gradient of images in scale space.

Tschumperle and Deriche proposed an anisotropic diffusion equation (11) for noise filtering and inpainting [7]. They proposed a vector-valued regularization equation, which makes use of structure tensor.

$$
\frac{\partial I_{i}}{\partial t}=\operatorname{trace}\left(\mathbf{T H}_{\mathbf{i}}\right)
$$

where $\mathbf{T}$ is the tensor field and $\mathbf{H}_{\mathbf{i}}$ is the Hessian matrix of the vector component $I_{i}$.

\section{NOISE REDUCTION BASED ON LDA AND DIFFUSION EQUATION}

LDA finds a direction for which the data are separated well [4]. It is also used in dimension reduction application in higher feature spaces [5]. We make use of LDA to generate a new image space, which is used for evaluating a common diffusion controlling term for each color channel. The regenerated space also has better characteristics for diffusion based filtering, because it emphasizes the differences between regions, which results in larger dynamic range.

We generate a new image space for diffusion controlling term by using gLDA and K-Means clustering algorithm recursively. All pixels in the image are grouped into two groups using K-Means clustering algorithm. The optimal direction vector, which separate the clustered data efficiently, is evaluated using regularized LDA algorithm. After finding the direction vector, each pixel data is moved along with the evaluated direction. The direction of each group is opposite, and the displacement is determined by the ratio of the size of each group and total number of pixels, and trace of each scatter matrix as follows.

$$
\begin{gathered}
\mathbf{d}_{\mathbf{i}}=2^{1+n_{i} / N} \times \operatorname{trace}\left(\mathbf{S}_{\mathbf{i}}\right) \mathbf{w}_{\mathbf{i}},(i=1,2) \\
L_{\mathbf{n}}= \begin{cases}0 & , \text { if } \sigma_{\mathbf{n}}^{2}<\epsilon \\
1 & , \text { otherwise }\end{cases} \\
\mathbf{x}_{\mathbf{n}}=\mathbf{x}_{\mathbf{n}}+\mathbf{d}_{\mathbf{i}} \times L_{\mathbf{n}}
\end{gathered}
$$

where $\mathbf{d}_{\mathbf{i}}$ is a displacement vector for a clustered group, and $\mathbf{x}_{\mathbf{n}}$ is a pixel data in it, which is a vector in $\mathbb{R}^{3} . L_{\mathbf{n}}$ is a local feature of each pixel to be robust to noise. In this paper, we use a simple local measure of variance of pixels within $3 \times 3$ 
windows where $\mathbf{x}_{\mathbf{n}}$ is located in the center, and $\epsilon$ is a threshold value. $N$ and $n_{i}$ are the total number of pixels in images and the number of data in group $i$. Fig. 1 illustrates this process conceptually. After moving the pixel data, the process is recursively done in each group respectively. When the variance or the size of each group reaches below the predefined threshold, the processing is stopped.

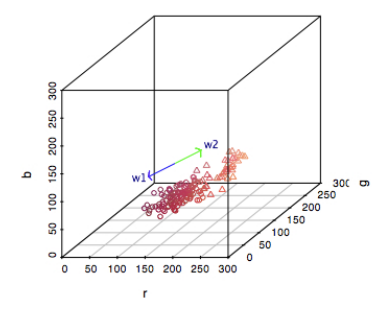

Fig. 1. Conceptual Illustration of Moving Pixel Data

The result of moving pixel data also has three dimensions. In order to reduce the dimensions without significant loss of information, we make use of PCA(Principle Component Analysis), which results in one dimensional data.

The diffusion controlling term is evaluated with the regenerated pixel data. First, we cluster pixel values within a fixed window into two groups with K-Means clustering algorithm. We model the difference between the center of each group as edge strength as follows.

$$
E S(i, j)=\left|c_{1}(i, j)-c_{2}(i, j)\right|
$$

where $c_{1}(i, j)$ represents the center of the first group, and the $c_{2}(i, j)$ is the center of the second group. After evaluating initial edge map, we apply noise filtering technique of eq. (10) to the initial edge map, which results in the filtered edge map.

$$
\frac{\partial v}{\partial t}=g|\nabla v| \nabla \cdot\left(\frac{\nabla v}{|\nabla v|}\right)-\lambda(1-g)(v-E)
$$

where $E$ means the initial edge map evaluated with eq. (15).

Finally, we use the extracted edge information from the initial image as a parameter of function of $g$ in eq. (10) instead of the magnitude of the smoothed gradient with Gaussian filtering.

$$
\frac{\partial u}{\partial t}=v|\nabla u| \nabla \cdot\left(\frac{\nabla u}{|\nabla u|}\right)-\lambda(1-v)(u-I)
$$

where $v=g(E S)=e^{\left(-\frac{E S^{2}}{K}\right)}$. As we mentioned before, the diffusion control term is evaluated in the beginning of filtering with an initial image once, which means that the evaluation of diffusion control term is not required in every iteration step. This edge map is used as the common diffusion controlling term for each color channel of the color image.

\section{EXPERIMENTAL RESULTS}

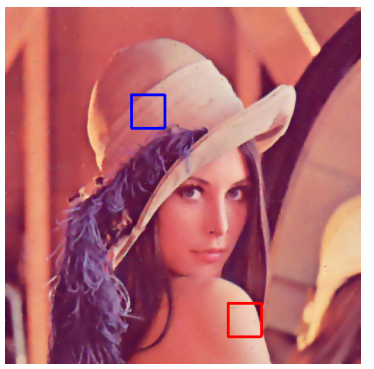

(a)

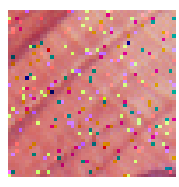

(c)

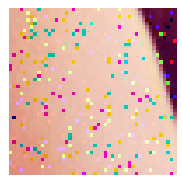

(f)

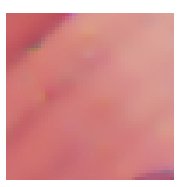

(d)

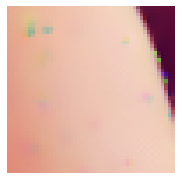

(g)

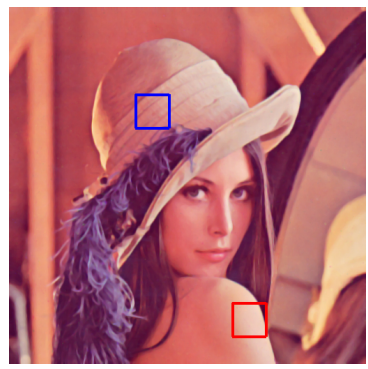

(b)

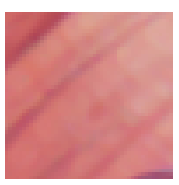

(e)

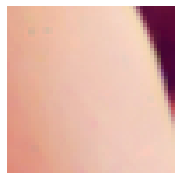

(h)
Fig. 2. Noise Filtering Results of Lena Image for Salt \& Pepper Noise. (a) is a filtering result of [7] , and (b) is a filtering result of the proposed method. (c) and (f) are the blue and the red rectangular regions of the noisy image. (d) and (g) are the result of [7]. (e) and (h) are the results of the proposed method.

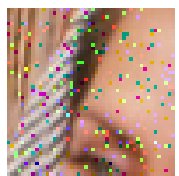

(a)

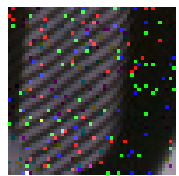

(d)

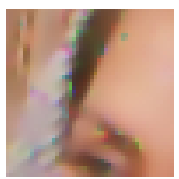

(b)

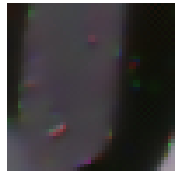

(e)

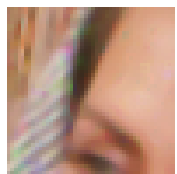

(c)

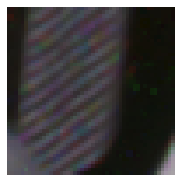

(f)
Fig. 3. Noise Filtering Results of Barbara Image for Salt \& Pepper Noise. (a) and (d) are noisy parts of the Barbara image, (b) and (e) are the results of [7], (c) and (f) are the results of our proposed method. 
Table 1. Comparison of PSNR(dB) Results

\begin{tabular}{|c|c|c|c|c|c|c|c|c|c|}
\hline & \multicolumn{3}{|c|}{ Gaussian Noise } & \multicolumn{3}{c|}{ Multiplicative Noise } & \multicolumn{3}{c|}{ Salt \& Pepper Noise } \\
\cline { 2 - 10 } & Lena & Pepper & Barbara & Lena & Pepper & Barbara & Lena & Pepper & Barbara \\
\hline Proposed Method & 36.65 & 36.81 & 35.26 & 34.25 & 36.01 & 32.80 & 35.23 & 35.05 & 31.15 \\
\hline Deriche [7] & 36.80 & 37.31 & 34.73 & 34.34 & 36.25 & 32.81 & 34.53 & 34.59 & 31.05 \\
\hline Silva [6] & 36.35 & 37.11 & 33.36 & 34.11 & 35.37 & 31.53 & 32.60 & 31.28 & 28.91 \\
\hline
\end{tabular}

In this section, we present results from experiment with our proposed method. Diffusion term in eq. (16) is calculated as follows [6].

$$
|\nabla u|\left(\nabla \cdot\left(\frac{\nabla u}{|\nabla u|}\right)\right)=\frac{u_{x}^{2} u_{y y}-2 u_{x} u_{y} u_{x y}+u_{y}^{2} u_{x x}}{u_{x}^{2}+u_{y}^{2}}
$$

where $u_{x}$ represents a partial derivative of $u$ with respect to $x$. (17) is approximation of a diffusion term with central difference and Euler's method. We use a fixed size window for K-Means clustering, which has a size $3 \times 3$.

To compare the performance of the proposed method, we evaluate PSNR (Peak Signal-to-Noise Ratio). The test images are degraded by additive Gaussian noise, multiplicative noise, and salt and pepper noise. (18) and (19) show the noise model of additive Gaussian noise and multiplicative noise respectively.

$$
\begin{gathered}
\mathscr{I}(x, y)=I_{o}(x, y)+\mathscr{N}(x, y) \\
\mathscr{I}(x, y)=I_{o}(x, y)+\mathscr{M} \times I_{o}(x, y)
\end{gathered}
$$

where $\mathscr{I}(x, y)$ is a noisy image data, $I_{o}(x, y)$ denotes the ideal image data without noise, and $\mathscr{N}(x, y)$ represents the signal independent additive Gaussian noise. Also $M$ is uniformly distributed random noise with mean 0 and a fixed variance. Typical test images such as Lena, Pepper, and Barbara are used in the experiment. Table 1 shows PSNR results, where the standard deviation of additive Gaussian noise is 10 , the variance for multiplicative noise is 0.02 , and the noise density of Salt \& Pepper noise is $4 \%$. Our proposed algorithm shows similar results in the point of PSNR as shown in Table1. However, as shown in Fig.2 and Fig.3, especially in the case of Salt \& Pepper noise, our method shows better visual quality than Tschumperle and Deriche's method [7].

\section{CONCLUSION}

In this paper, we have presented a new noise filtering method using diffusion equation and LDA. We make a different approach to evaluate diffusion controlling term. The new image space is generated by recursive LDA and K-Means clustering algorithm with local feature measure. It is used to evaluate edge map, which is used as a diffusion control term. The evaluated edge map is used as the common diffusion controlling term for noise filtering of a color image. Experimental results shows that our proposed method preserves image features and reduces noise such as additive Gaussian noise, multiplicative noise, and Salt \& Pepper noise well and better results in the point of image quality.

\section{REFERENCES}

[1] Fietro Perona and Jitendra Malik, "Scale-space and edge detection using anisotropic diffusion," IEEE Transactions on Pattern Analysis and Machine Intelligence, vol. 12, no. 7, pp. 629-639, July 1990.

[2] K.Niklas Nordström, "Biased anisotropic diffusion - a unified regularization and diffusion approach to edge detection," Image Vis. Comput., , no. 8, pp. 318-327, 1990.

[3] Joachim Weickert, "Multiscale texture enhancement," in Computer Analysis of Images and Patterns: LNCS in Computer Science, Berlin, 1995, vol. 970, pp. 230-237, Springer.

[4] Richard O.Duda, Peter E. Hart, and David G.Stork, Pattern Classification, A Wiley-Interscience Publication, 2000 .

[5] Cheonghee Park and Haesun Park, "A comparison of generalized lda algorithms for undersampled problems," Tech. Rep., University of Minnesota, Computer Science and Engineering, December 2003.

[6] C. A. Z. Barcelos, M. Boaventura, and Jr. Silva, E. C., “A well-balanced flow equation for noise removal and edge detection," IEEE Transactions on Image Processing, vol. 12, no. 7, pp. 751-763, July 2003.

[7] D. Tschumperle and R. Deriche, "Vector-valued image regularization with pdes: a common framework for different applications," Transactions on Pattern Analysis and Machine Intelligence, vol. 27, no. 4, pp. 506-517, 2005. 